\title{
Impact of HIV/Aids on Child Mortality before the Highly Active Antiretroviral Therapy Era: A Study in Pointe-Noire, Republic of Congo
}

\author{
Camille Lallemant, ${ }^{1}$ Gaston Halembokaka, ${ }^{2}$ Gaelle Baty, ${ }^{3}$ Nicole Ngo-Giang-Huong, \\ Francis Barin, ${ }^{3}$ and Sophie Le Coeur ${ }^{5}$ \\ ${ }^{1}$ Brighton and Sussex Medical School, University of Sussex, Brighton, East Sussex, BN1 9PX, UK \\ ${ }^{2}$ Association Congolaise pour la Santé Publique et Communautaire, Pointe-Noire, Democratic Republic of Congo \\ ${ }^{3}$ Laboratoire de Virologie, EA 3856 and Centre National de référence du VIH, CHU Bretonneau, François Rabelais University, \\ 2 bd Tonnellé, 37044 Tours, France \\ ${ }^{4}$ Institut de Recherche pour le Développement, UMI 174/Faculty of Associated Medical Sciences, Chiang Mai University, \\ 187/10 Changklan Rd., Changklan, Muang, Chiang Mai 50100, Thailand \\ ${ }^{5}$ UMR 196 CEPED, Université Paris-Descartes, INED, IRD, 19 rue Jacob, 75006 Paris, France
}

Correspondence should be addressed to Sophie Le Coeur, lecoeur@ined.fr

Received 5 March 2010; Accepted 15 July 2010

Academic Editor: Blaise Genton

Copyright (C) 2010 Camille Lallemant et al. This is an open access article distributed under the Creative Commons Attribution License, which permits unrestricted use, distribution, and reproduction in any medium, provided the original work is properly cited.

Few studies have documented the contribution of HIV/AIDS to mortality among children under 15 years. From June 30 to October 19,2001 , all child deaths $(n=588)$ registered to the morgue and/or hospitals of the city of Pointe-Noire, Congo, were investigated using a combined approach including an interview of relatives and postmortem clinical and biological HIV diagnosis. Twenty-one percent of children were HIV positive, while $10.5 \%$ of deaths were attributed to AIDS. The most common causes of death in HIVinfected children were pneumonia (30\%), pyrexia (22\%), diarrhoea (16\%) and wasting syndrome (16\%). Infant mortality rate was estimated 6.3 times higher in children born to HIV-infected mothers compared to HIV-uninfected mothers. This study provides a direct measure of HIV/AIDS as impact on child mortality using a rapid and reliable method. A significant number of deaths could be prevented if HIV infection was diagnosed earlier and infants were provided with antiretroviral treatments.

\section{Introduction}

In 2007, UNAIDS estimated that 2.0 million children under 15 years were living with HIV/AIDS, among whom $90 \%$ were in sub-Saharan Africa and that during the same year 270,000 children had died of AIDS [1].

In developing countries, the relative importance of AIDS among all causes of death in children is difficult to evaluate because death registers are often incomplete, and causes of death are often imprecise or inaccurate [2]. In addition, the diagnosis of AIDS is particularly difficult in children since the signs and symptoms are not specific [3]. Also, physicians and families may be reluctant to declare HIV/AIDS as the cause of death, a disease which remains highly stigmatized. Finally, when an HIV serology is available, it may only reflect the HIV status of the mothers in children less than 15 months due to the transfer of anti-HIV antibodies through the placenta [4]. Although the Republic of the Congo was one of the first countries in Africa to be affected by the HIV epidemic, HIV prevalence in the adult population has remained relatively stable over the past 25 years. In 2001, it was estimated as $4.4 \%$ in adults [1]. A small cohort study carried out during the first decade of the epidemic showed that infant mortality in children born to HIV-infected mothers exceeded 260 per thousand, while it was only 44 per thousand in children born to HIV-negative mothers [5]. As the definitive diagnosis of $\mathrm{HIV}$ in infants was not possible at the time, it is possible that this excess of mortality reflected both children's HIV 
infection as well as the impact of AIDS in their family. In order to more precisely investigate the relative contribution of HIV/AIDS to the under-15 mortality, we have analysed the causes of all deaths in children reported in the city of PointeNoire, Congo, using an approach combining verbal autopsy, clinical and biological data.

\section{Methods}

2.1. Survey. From June 30 to October 18, 2001, all deaths occurring in the city of Pointe-Noire were investigated. Physicians were posted at the morgue to examine the bodies following a standardised procedure, recorded signs of AIDS, based on the WHO clinical definition, and obtain a blood sample by intracardiac puncture [6]. They also interviewed the individual bringing the body to the morgue, usually a family member, on the circumstances of death.

For all cases previously hospitalized before death, the hospital files were reviewed in order to obtain further clinical and biological information about the cause of death. Almost all medical facilities were visited including the two main public hospitals, the military hospital, 7 private clinics, 13 health centers, the Center for endemic diseases, and the HIV clinic.

The admission files of all these hospitals and other health centers were also systematically reviewed to search for additional deaths not registered at the morgue, and information was collected about these deaths.

2.2. HIV Test. Plasma samples were tested for HIV antibodies by indirect enzyme immunoassay (HIV-1/HIV-2 Abcapture ELISA, Ortho diagnostic Systems Inc, Raritan, New Jersey, USA) and confirmed by serotyping [7]. For infants less than 18 months with either positive or weakly positive HIV-1 serology, an HIV DNA real-time PCR test was performed on dried blood spots, in order to rule out passive antibody carriage [8]. All the biological tests were performed blindly.

2.3. Causes of Death. The causes of death were coded by an independent physician, taking into account the information available from the family interview, postmortem examination and/or hospital file. They were classified according to the WHO 10th International Classification of Diseases and were grouped into 12 major headings [9].

Deaths were attributed to AIDS when the HIV serologyand PCR for infants aged less than 18 months-was positive and at least one major and one minor criteria of the WHO clinical case definition were present at examination-major sign: severe wasting; minor signs: lesions from generalized pruritic dermatitis, scars of herpes zoster, oral candidiasis, and generalized lymphadenopathy; major symptoms: chronic diarrhea or fever for more than one month [10]. If the HIV serology was not available, AIDS was considered as the cause of death when in addition to these clinical criteria, AIDS, retroviral disease, immunodeficiency, or HIV, was mentioned as the cause of death at the hospital. Because
AIDS is generally the underlying cause of death, the primary cause of death in HIV-infected children was also determined.

2.4. Infant Mortality Estimates. To estimate the infant mortality rate, the number of deaths was reported to the number of live births, obtained from an enumeration of all live and still births carried out in the six public and nine private maternity clinics of the city, during the study period [6]. It should be noted that, in the Republic of the Congo, almost all women give birth in a maternity clinic, and in the rare event of a woman delivering at home, her newborn should be registered shortly after birth at a maternity clinic in order to avoid a fine. Infant mortality rates according to the HIV status of the mother were also estimated using the HIV prevalence rate of $6.3 \%$ obtained from an anonymous seroprevalence survey in all delivering women, carried out in $2002[11]$.

2.5. Statistical Analysis and Ethical Approval. Statistics were performed on SPSS version 14.0. The normality of the age distribution was tested using the Kolmogorov-Smirnov Test. Because it was not normally distributed, a nonparametric test, the Wilcoxon Rank Sum test, was used to compare the medians.

The protocol was approved scientifically and ethically by the Ministry of Health of the Republic of Congo. All samples and questionnaires were coded, and personal identifications were discarded.

\section{Results}

3.1. Number of Deaths. During the study period, a total of 588 child deaths were recorded, including 365 admitted to the morgue and 233 identified through hospital admission files. The distribution of all 588 deaths by age and sex is provided in Table 1 . The sex ratio was 1.02 . The median age at death was 12.5 months (Interquartile range $(\mathrm{IQR})=1.1-$ 31 ). Overall, out of the 588 child deaths, $75 \%$ had died in a health care setting, $18 \%$ at home, $5 \%$ on the way to the hospital, and $2 \%$ in a public place.

3.2. HIV Prevalence. Among the 365 children admitted to the morgue, only 5 (1.4\%) could not be tested for HIV because either the blood sample could not be drawn due to body damage or severe bleeding, or the sample was insufficient. The HIV serology was positive in 77 of all children (21.1\%). An HIV-DNA PCR was performed on all samples from children under 18 months of age $(n=28)$ who had a positive or borderline HIV serology test. Among them, 3 children had a negative PCR result and were considered as uninfected. Thus, a total of 74 children (20.6\%) were found to be HIV infected.

Table 2 provides the HIV infection prevalence according to age. It was highest, $31 \%$, in the $0-1$ year-age group. The median age at death in HIV-infected children was 24.5 months (IQR: 5.4-53.7 months) similar to the median age at death in HIV-uninfected children, 24.4 months (IQR: 12.348.8) $(P=.267)$. 
TABle 1: Age distribution of deceased children $(n=588)$.

\begin{tabular}{|c|c|c|c|c|c|c|}
\hline \multirow{2}{*}{ Age groups } & \multicolumn{2}{|c|}{ Boys } & \multicolumn{2}{|c|}{ Girls } & \multicolumn{2}{|c|}{ Total } \\
\hline & $N$ & $\%$ & $N$ & $\%$ & $n$ & $\%$ \\
\hline 0-11 months & 144 & 49.5 & 124 & 41.8 & 268 & 45.5 \\
\hline $1-4$ years & 104 & 35.7 & 128 & 43.1 & 232 & 39.5 \\
\hline $5-9$ years & 23 & 7.9 & 25 & 8.4 & 48 & 8.2 \\
\hline 10-14 years & 20 & 6.9 & 20 & 6.7 & 40 & 6.8 \\
\hline Total & 291 & 100.0 & 297 & 100.0 & 588 & 100.0 \\
\hline
\end{tabular}

TABLE 2: HIV infection rate according to the age of the deceased children.

\begin{tabular}{lccc}
\hline \multirow{2}{*}{ Age groups } & Deaths & Total & \\
& $N$ & HIV+ & $\%$ \\
\hline $0-11$ months & 91 & 28 & 30.8 \\
1-4 years & 189 & 30 & 15.9 \\
5-14 years & 80 & 16 & 20.0 \\
\hline Total & 360 & 74 & 20.6 \\
\hline
\end{tabular}

3.3. Causes of Death. Table 3 provides the causes of death according to age in all children $(n=588)$, whether or not they were brought to the morgue. Among infants, neonatal death was the cause of half of the deaths, with AIDS accounting for $7.5 \%$. In children aged 1 to 4 years, malaria was the cause of $36 \%$ of deaths, followed by measles in $21 \%$ of cases. AIDS accounted for $12 \%$ of deaths in this age group. Finally, in children above 5 years, AIDS and malaria were the leading causes of death, both accounting for $17 \%$ of deaths, respectively.

For the 74 children with confirmed HIV-infection, the most frequent causes of death declared by the family were respiratory infection (28\%), malaria (23\%), and chronic diarrhoea and/or fever $(15 \%)$. In one case only did the family mention AIDS as the cause of death. Only 53 of these children (71.6\%) fulfilled the clinical AIDS case definition. Finally, the primary cause of death in these HIV-infected children were bronchopneumonia in $30 \%$, pyrexia in $22 \%$, diarrhoea in 16\%, and wasting syndrome in 16\% (Table 4). During the first year of life, bronchopneumonia was the cause of more than $50 \%$ of the deaths in HIV-infected children.

3.4. Infant Mortality Estimates. Reporting the number of death under 1 year to the number of live-births during the study period, the overall infant mortality rate was estimated at 44 per 1000 (95\% CI: 39-50) (Table 5). In the study population, the percentage of HIV-infected children was $30.8 \%$ (28/91) among those under 1 year of age who could be tested. Assuming that the rate of infection was the same in children who could not be tested, the number of HIV positive and negative deaths could be estimated at 82 and 186, respectively. Using the HIV prevalence rate of $6.3 \%$ (95\% CI: $5.0 \%-7.8 \%$ ) obtained in an anonymous survey of all women who had given birth in all maternity units of the city between September 27 and October 19, 2002 [6], the mortality rates in children born to HIV-positive mothers and HIV-negative mothers could be estimated at 209 per thousand (95\% CI: 170-253) and 33 per thousand (95\% CI: 28-38), respectively. Therefore, the observed risk of death during their first year of life was found to be 6.3 times higher in infants born to HIV-positive mothers as compared to HIVnegative mothers.

\section{Discussion}

This study evaluates, in a large central African city, the contribution of HIV/AIDS to mortality in children less than 15 years of age, relatively to other causes of death before the Highly Active Antiretroviral (HAART) era. The most common immediate causes of death in HIV-infected children were pneumonia (30\%), pyrexia (22\%), diarrhoea $(16 \%)$, and wasting syndrome $(16 \%)$. We estimated that infant mortality rate among HIV exposed children was 6.3 times higher than that among children born to HIV-uninfected mothers.

Clinical AIDS was found to be the cause of $10.5 \%$ of deaths, while $20 \%$ of the children tested were found to be HIV-infected. Indeed, it is likely that when the HIV test was not available, the clinical approach for diagnosis underestimated the number of AIDS cases. The fact that only $72 \%$ of the HIV-infected children fulfilled the AIDS case definition confirms the lack of sensitivity of the clinical diagnosis of AIDS [3].

The prevalence of HIV infection was highest (31\%) in the 0-1 year age group reflecting the high level of mortality during the first year of life in perinatally infected children, in the absence of antiretroviral treatment. In our study, more than $50 \%$ of the HIV-infected infants died of pneumonia. It is very likely that their mothers were not aware of their HIV status. At the time, HIV testing during pregnancy was not systematically offered. If Co-trimoxazole had been systematically provided to all children born to HIVinfected mothers, mortality related to pneumocystis jiroveci pneumonia would have been drastically reduced [12].

The fact that the families mentioned AIDS as the cause of death in only one case demonstrates a high level of denial, even in a country where the HIV epidemic started more than 25 years ago. Despite decades of information and prevention campaigns, HIV/AIDS remains highly stigmatized.

Some factors which are generally associated with infant mortality were not investigated. For example, the maternal 
TABLE 3: Causes of deaths according to the age of the children $(n=588)$.

\begin{tabular}{|c|c|c|c|c|c|c|c|c|}
\hline \multirow{2}{*}{ Causes of death } & \multicolumn{2}{|c|}{ 0-11 months } & \multicolumn{2}{|c|}{$1-4$ years } & \multicolumn{2}{|c|}{ 5-14 years } & \multicolumn{2}{|c|}{ Total } \\
\hline & $N$ & $\%$ & $N$ & $\%$ & $N$ & $\%$ & $N$ & $\%$ \\
\hline Neonatal deaths & 137 & 51.1 & 0 & 0.0 & 0 & 0.0 & 137 & 23.3 \\
\hline Malaria & 18 & 6.7 & 83 & 35.8 & 15 & 17.0 & 116 & 19.7 \\
\hline Measles & 12 & 4.5 & 49 & 21.1 & 6 & 6.8 & 67 & 11.4 \\
\hline AIDS & 20 & 7.5 & 27 & 11.6 & 15 & 17.0 & 62 & 10.5 \\
\hline Respiratory diseases & 30 & 11.2 & 19 & 8.2 & 6 & 6.8 & 55 & 9.4 \\
\hline Digestive diseases & 24 & 9.0 & 16 & 6.9 & 6 & 6.8 & 46 & 7.8 \\
\hline Infectious diseases & 9 & 3.4 & 14 & 6.0 & 9 & 10.2 & 32 & 5.4 \\
\hline Anemia & 9 & 3.4 & 11 & 4.7 & 7 & 8.0 & 27 & 4.6 \\
\hline Accidents & 2 & 0.7 & 4 & 1.7 & 7 & 8.0 & 13 & 2.2 \\
\hline Other noninfectious diseases & 2 & 0.7 & 2 & 0.9 & 9 & 10.2 & 13 & 2.2 \\
\hline Indeterminate & 2 & 0.7 & 5 & 2.2 & 6 & 6.8 & 13 & 2.2 \\
\hline Malnutrition & 3 & 1.1 & 2 & 0.9 & 2 & 2.3 & 7 & 1.2 \\
\hline Total & 268 & 100.0 & 232 & 100.0 & 88 & 100.0 & 588 & 100.0 \\
\hline
\end{tabular}

Table 4: Primary causes of death in children with confirmed HIV infection, according to age $(n=74)$.

\begin{tabular}{|c|c|c|c|c|c|c|c|c|}
\hline \multirow{2}{*}{ Primary causes of death } & \multicolumn{2}{|c|}{$0-11$ months } & \multicolumn{2}{|c|}{$1-4$ years } & \multicolumn{2}{|c|}{ 5-14 years } & \multicolumn{2}{|c|}{ Total } \\
\hline & $N$ & $\%$ & $N$ & $\%$ & $N$ & $\%$ & $N$ & $\%$ \\
\hline Bronchopneumonia & 15 & 54 & 4 & 13 & 3 & 19 & 22 & 30 \\
\hline Pyrexia & 2 & 7 & 12 & 40 & 2 & 12 & 16 & 22 \\
\hline Diarrhea & 6 & 21 & 3 & 10 & 3 & 19 & 12 & 16 \\
\hline Wasting syndrome & 2 & 7 & 4 & 13 & 6 & 38 & 12 & 16 \\
\hline Measles & 3 & 11 & 5 & 17 & 2 & 12 & 10 & 14 \\
\hline Anemia & 0 & 0 & 1 & 3 & 0 & 0 & 1 & 1 \\
\hline Other non-infectious & 0 & 0 & 1 & 3 & 0 & 0 & 1 & 1 \\
\hline Total & 28 & 100 & 30 & 100 & 16 & 100 & 74 & 100 \\
\hline
\end{tabular}

vital status was not systematically recorded. Therefore, it was not possible to assess the relationship between the child death and its mother's death. Also, in Pointe-Noire, the majority of mothers are breastfeeding their infants until at least 12 months of age. Since infants mode of feeding was not documented in our survey, we cannot ascertain the contribution of lack or improper breastfeeding as an indirect cause of infants death from diarrhoea or malnutrition.

In this study, the percentages of neonatal death $(23.3 \%)$, malaria (19.7\%), and AIDS deaths (10.5\%) in children are consistent with the results from a similar study performed in Brazzaville, the capital city of the Republic of Congo, in 1998 [13]. They accounted, respectively, for $26.0 \%, 14.9 \%$ and $10.0 \%$ of under-fifteen mortality. However, in Pointe noire, the percentage of death due to measles $(11.4 \%)$ was much higher than in Brazzaville where it was only $4.1 \%$. Indeed the Pointe-Noire study was carried out during the dry season during a measles epidemic breakout, shortly after the end of the 1999 civil war during which immunisation effort had been lessened.

In a hospital-based study in South Africa, the main causes of under-five child mortality were lower respiratory tract infections (31.4\%), AIDS (21.3\%), and sepsis (13.4\%) [14]. Also, in a population-based study in South Africa, the main causes of death established by verbal autopsy in children under the age of 15 were AIDS (39\%), respiratory diseases (23\%), and neonatal deaths (7\%) [15]. The lower proportion of AIDS deaths in our study is probably related to the lower HIV prevalence rate in the general population in the Congo [1].

The overall infant mortality rate derived from this study, 44 per 1000, is probably underestimated. Indeed, figures provided for 2000-2005 by the United Nation, are higher, at 75.1 per thousand [16]. In Congolese culture, ancestor/dead worship is essential and requires ostentatious funerals [17]. For children, however, the cultural need for ritual funeral is less important, and, because of its high costs, parents may try to avoid reporting their child's death, particularly neonates and infants. Thus, it is likely that additional children, especially small infants, may have died at home and be buried clandestinely. Other potential biases related to under-registration of deaths, could be due to migration, seasonal mortality pattern, or census inaccuracy.

The estimation of the mortality rate in infants born to HIV-positive mothers (209 per 1000) can be compared with estimations from studies where infants born from HIVinfected mothers were followed prospectively from birth. The mortality rate is slightly lower than in one of these 
TABLE 5: Mortality rates in children less than 1 year according to the HIV status of their mothers.

\begin{tabular}{lcccc}
\hline & Total & $\begin{array}{c}\text { HIV+ } \\
\text { mothers }\end{array}$ & $\begin{array}{c}\text { HIV }- \\
\text { mothers }\end{array}$ & $\begin{array}{c}\text { Rate ratio } \\
\text { HIV+/HIV- }\end{array}$ \\
\hline Number of live births & 6025 & 392 & 5633 & 186 \\
Number of deaths $<1$ year & 268 & 82 & 33 & - \\
Infant mortality rate (per 1000) & 44 & 209 & $28-38$ & 6.3 \\
$95 \%$ CI & $39-50$ & $170-253$ & - \\
\hline
\end{tabular}

studies in Brazzaville, 260 per 1000 [5]. The lower estimation of the infant mortality observed in a pooled analysis of 9 HIV perinatal clinical trials in Sub-Saharan Africa, 110 per 1000 [18], is most probably due to the health care support provided within clinical trial settings.

The infant mortality rate in children born to HIVinfected mothers and HIV-uninfected mothers, obtained in our study, 209 per 1000 and 33 per 1000, respectively, can also be compared with the rates estimated from rural populationbased surveys where mothers had been tested for HIV. These rates were 225 per 1000 and 53 per 1000, respectively, in a large population study in Uganda [19], 158 per 1000 and 79 per 1000, respectively, in a study in Tanzania [20], and 331 per 1000 and 115 per 1000 in a study in Malawi [21]. The differences are probably related to the level of overall child mortality, which is lower in an urban setting such as PointeNoire, and, as mentioned earlier, a potential underreporting of infant deaths.

Yet, because underreporting of infant mortality should not differ according to the HIV status of the parents, the rate ratio of mortality between infants born to HIV-infected and HIV-uninfected mothers should not be affected. The 6.3 times higher risk of death in infants born to HIV-positive mothers as compared to HIV-negative mothers is consistent with the results of the Brazzaville study where it was 6.2 times higher [5]. It is, however, higher than in the populationbased studies in Uganda [19] and Tanzania [20] where they were 4.2 and 2.9, respectively. This could be explained by a difference in mother to child HIV transmission rate or a higher sensitivity of the diagnosis of AIDS as a cause of death in our study in the Republic of Congo.

Our diagnosis procedure based on family interviews, postmortem examination, review of hospital records, and biological tests provides reliable estimates of the percentage of death related to HIV/AIDS, in particular for children admitted to the morgue for whom only $1.4 \%$ could not be tested for HIV. In addition, the use of PCR testing in children younger than 18 months allowed us to exclude uninfected children born to HIV-infected mothers.

\section{Conclusion}

This study provides a direct measure of HIV/AIDS' impact on child mortality relative to other causes, using a relatively rapid and reliable method.

Our study, performed before the HAART era, shows that $50 \%$ of the deaths in children were attributable to measles, malaria, or diarrhea, all preventable deaths. It remains essen- tial to reinforce the existing children public health programs such as immunization, breastfeeding promotion, malaria control through impregnated bed nets, and provision of oral rehydration.

In the past 15 years, the provision of simple antiretroviral regimens during pregnancy have been shown to dramatically reduce the risk of transmission of HIV from mother to child $[22,23]$. The systematic provision of HIV testing and antiretrovirals for HIV-infected women during pregnancy would therefore dramatically reduce the overall child mortality from AIDS. Furthermore, as recently demonstrated in the CHER study showing a 76\% reduction of early infant mortality in children receiving HAART within the first year of life independently of their clinical or immunological condition, early HIV diagnosis followed by HAART provision would considerably reduce the risk of mortality of HIV-infected children [24].

\section{Acknowledgments}

The paper was supported by the Institut National d'Etudes Démographiques, the Ministère des Affaires Etrangères, the Institut de Recherche pour le Développement, France, and UNAIDS Intercountry team for West and Central Africa.

\section{References}

[1] UNAIDS/WHO, "Report on the global AIDS epidemic [electronic resource]. Promotion," http://www.unaids.org/.

[2] J. Stover, P. D. Ghys, and N. Walker, "Testing the accuracy of demographic estimates in countries with generalized epidemics," AIDS, vol. 18, no. 2, pp. S67-S73, 2004.

[3] C. L. van Gend, M. L. Haadsma, P. J. J. Sauer, and C. J. Schoeman, "Evaluation of the WHO clinical case definition for pediatric HIV infection in Bloemfontein, South Africa," Journal of Tropical Pediatrics, vol. 49, no. 3, pp. 143-147, 2003.

[4] J. Lujan-Zilbermann, C. A. Rodriguez, and P. J. Emmanuel, "Pediatric HIV infection: diagnostic laboratory methods," Fetal and Pediatric Pathology, vol. 25, no. 5, pp. 249-260, 2006.

[5] M. Lallemant, S. Le Coeur, D. Cheynier et al., "Motherchild transmission of HIV-1 and infant survival in Brazzaville, Congo," AIDS, vol. 3, no. 10, pp. 643-646, 1989.

[6] S. Le Coeur, G. Halembokaka, M. Khlat et al., "Impact of AIDS on adult mortality: a morgue-based study in Pointe-Noire, Republic of Congo," AIDS, vol. 19, no. 15, pp. 1683-1687, 2005.

[7] F. Barin, Y. Lahbabi, L. Buzelay et al., "Diversity of antibody binding to V3 peptides representing consensus sequences 
of HIV type 1 genotypes A to E: an approach for HIV type 1 serological subtyping," AIDS Research and Human Retroviruses, vol. 12, no. 13, pp. 1279-1289, 1996.

[8] M. Burgard, M. L. Chaix, N. Ngo-Giang-Huong, M. Leruez, and C. Rouzioux, "Real-time HIV-1 PCR for the diagnosis of infection in newborns of African mothers," in Proceedings of the 8th Conference on Retroviruses and Opportunistic Infections, Chicago, Ill, USA, February 2001.

[9] World Health Organization, ICD-10: International Statistical Classification of Diseases and Related Health Problems, World Health Organization, Geneva, Switzerland, 10th edition, 19921994.

[10] World Health Organisation, "W.H.O./CDC case definition for AIDS," Weekly Epidemiological Record, vol. 61, no. 10, pp. 6976, 1986.

[11] S. Le Coeur, M. Khlat, G. Halembokaka et al., "HIV and the magnitude of pregnancy-related mortality in Pointe Noire, Congo," AIDS, vol. 19, no. 1, pp. 69-75, 2005.

[12] C. Chintu, G. J. Bhat, A. S. Walker et al., "Co-trimoxazole as prophylaxis against opportunistic infections in HIV-infected Zambian children (CHAP): a double-blind randomised placebo-controlled trial," The Lancet, vol. 364, no. 9448, pp. 1865-1871, 2004.

[13] G. Pictet, S. Le Coeur, P. M'Pelé, N. Brouard, and M. Lallemant, "Contribution of AIDS to the general mortality in Central Africa: evidence from a morgue-based study in Brazzaville, Congo," AIDS, vol. 12, no. 16, pp. 2217-2223, 1998.

[14] A. Krug, M. Patrick, R. C. Pattinson, and C. Stephen, "Childhood death auditing to improve paediatric care," Acta Paediatrica, vol. 95, no. 11, pp. 1467-1473, 2006.

[15] A. Garrib, S. Jaffar, S. Knight, D. Bradshaw, and M. L. Bennish, "Rates and causes of child mortality in an area of high HIV prevalence in rural South Africa," Tropical Medicine and International Health, vol. 11, no. 12, pp. 1841-1848, 2006.

[16] United Nation. Department of Economic and Social Affairs. Population Division. World Mortality 2007.

[17] F. Hagenbucher-Sacripanti, Santé et Rédemption par les Génies au Congo, Publisud, Paris, France, 1992.

[18] M.-L. Newell, H. Coovadia, M. Cortina-Borja, N. Rollins, P. Gaillard, and F. Dabis, "Mortality of infected and uninfected infants born to HIV-infected mothers in Africa: a pooled analysis," The Lancet, vol. 364, no. 9441, pp. 1236-1243, 2004.

[19] J. S. Nakiyingi, M. Bracher, J. A. G. Whitworth et al., "Child survival in relation to mother's HIV infection and survival: evidence from a Ugandan cohort study," AIDS, vol. 17, no. 12, pp. 1827-1834, 2003.

[20] J. Ng'weshemi, M. Urassa, R. Isingo et al., "HIV impact on mother and child mortality in rural Tanzania," Journal of Acquired Immune Deficiency Syndromes, vol. 33, no. 3, pp. 393404, 2003.

[21] A. C. Crampin, S. Floyd, J. R. Glynn et al., "The long-term impact of HIV and orphanhood on the mortality and physical well-being of children in rural Malawi," AIDS, vol. 17, no. 3, pp. 389-397, 2003.

[22] M. Lallemant, G. Jourdain, S. Le Coeur et al., "Single-dose perinatal nevirapine plus standard zidovudine to prevent mother-to-child transmission of HIV-1 in Thailand," The New England Journal of Medicine, vol. 351, no. 3, pp. 217-228, 2004.

[23] F. Dabis, L. Bequet, D. K. Ekouevi, et al., "Field efficacy of zidovudine, lamivudine and single dose nevirapine to prevent peripartum HIV transmission," AIDS, vol. 19, no. 3, pp. 309318, 2005.

[24] A. Violari, M. F. Cotton, D. M. Gibb et al., "Early antiretroviral therapy and mortality among HIV-infected infants," The New England Journal of Medicine, vol. 359, no. 21, pp. 2233-2244, 2008. 


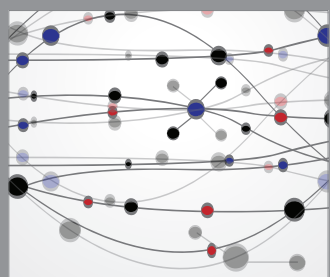

The Scientific World Journal
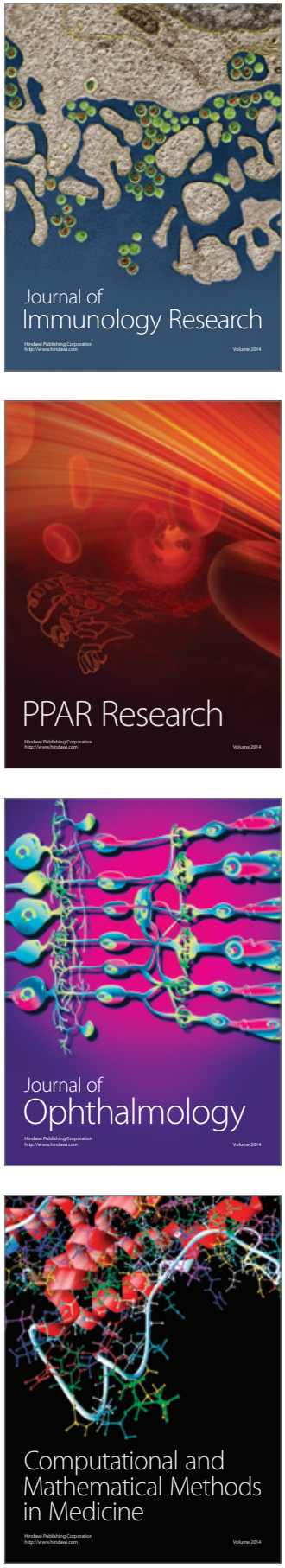

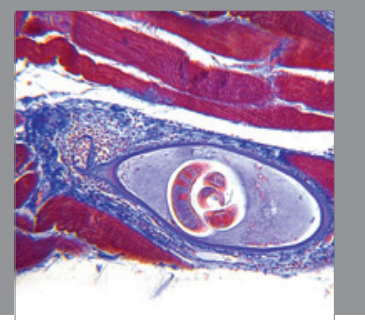

Gastroenterology

Research and Practice
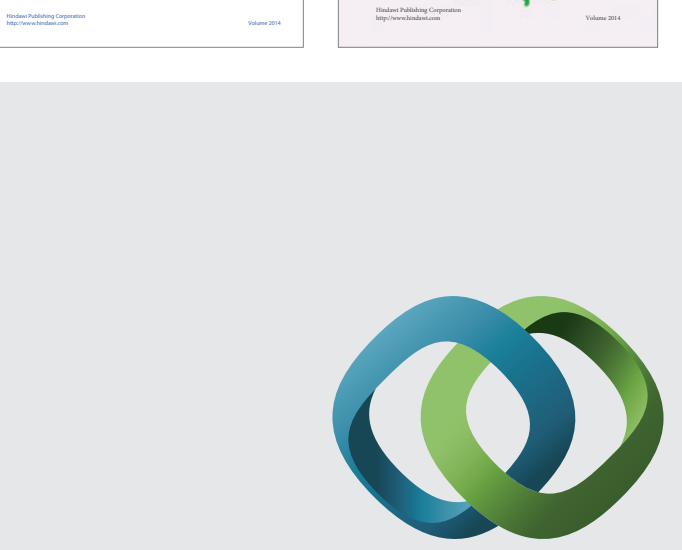

\section{Hindawi}

Submit your manuscripts at

http://www.hindawi.com
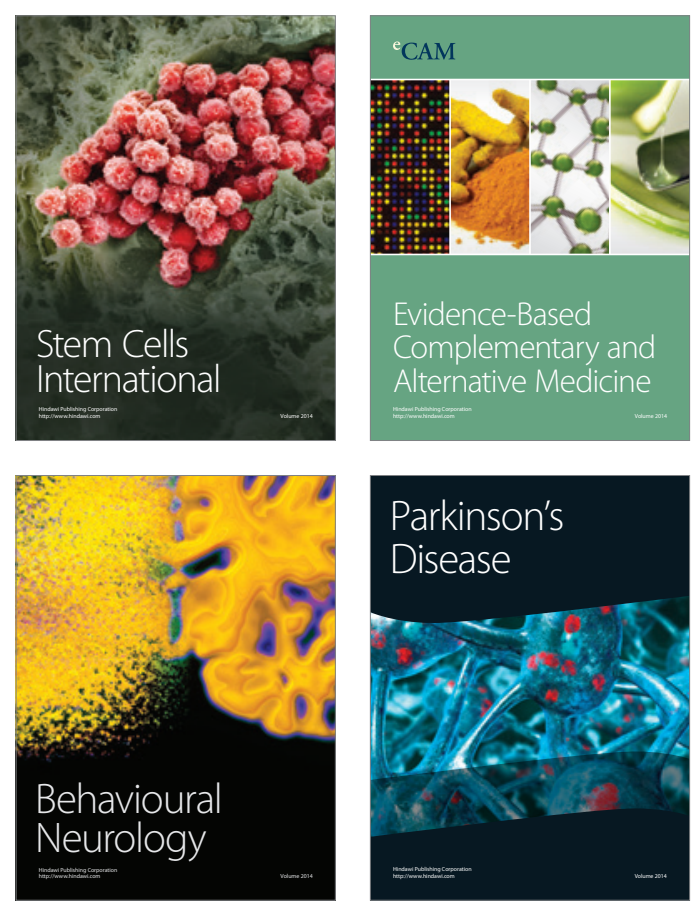

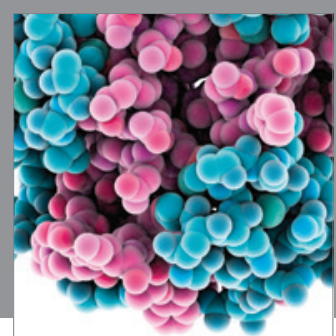

Journal of
Diabetes Research

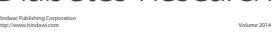

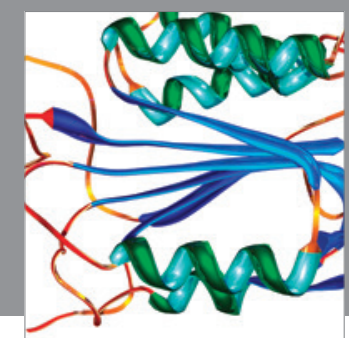

Disease Markers
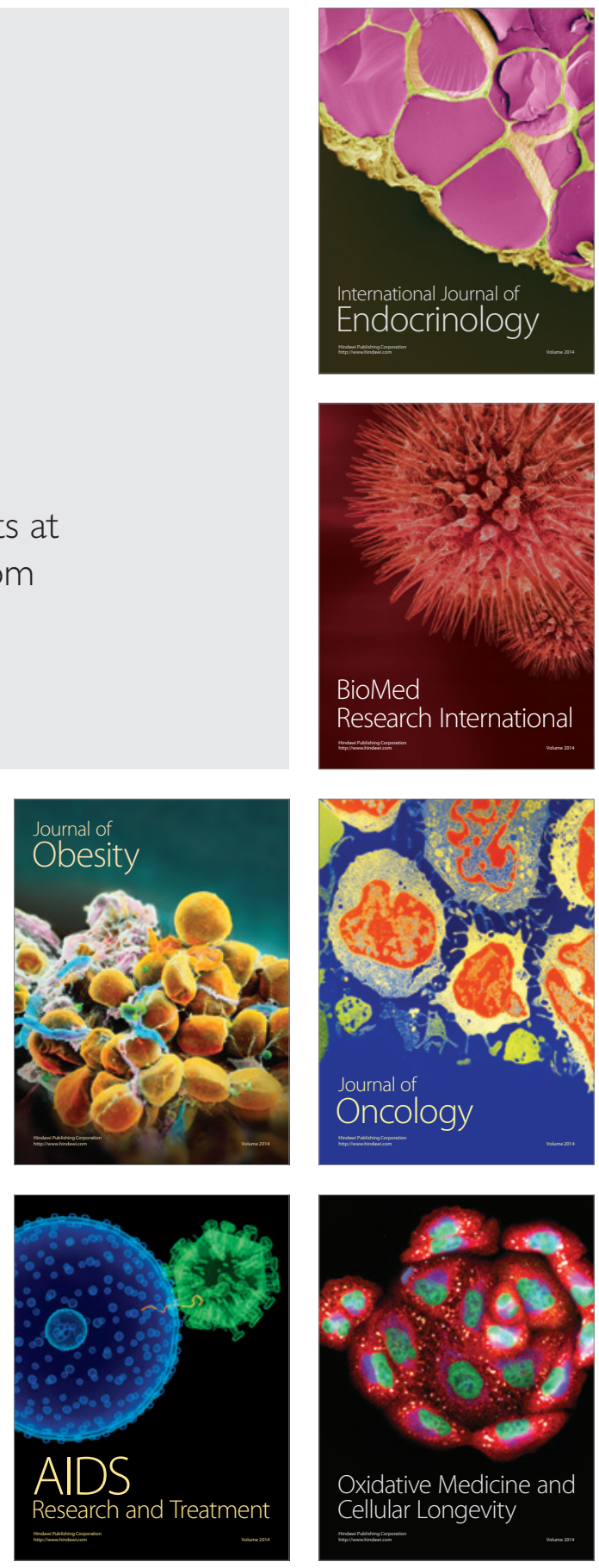